\title{
A Review of Optimal maintenance scheduling of generators using PSO technique
}

\author{
Priyanka B. Pagare ${ }^{1}$, Ganesh G. Mhatre ${ }^{2}$ \\ Student, Electrical Engineering Department, Yadavrao Tasgaonkar Institute of Engg., and Technology, Karjat, India ${ }^{1}$ \\ Asst. Professor, Electrical Engineering Department, NHITM College, Thane, India ${ }^{2}$
}

\begin{abstract}
This paper illustrates use of particle swarm optimization technique for effective and optimal maintenance scheduling of generating units ensuring economical and reliable operation of power system. Initially problems associated with the generator maintenance scheduling in modern power systems are illustrated, also explaining the need and importance of generator maintenance. It briefly describes the maintenance scheduling of generating units by application of PSO technique. This paper proposes a particle swarm optimization algorithm satisfying the system load demand and crew constraints. This technique converges to the optimal solution where most analytical methods fail to converge by generating schedules with high system reliability indices which can be represented by generation, crew, fitness and reliability plots.
\end{abstract}

Keywords: Generator maintenance schedule (GMS), optimization, personal best (pbest), global best (gbest), Particle swarm optimization (PSO) Technique and System reliability.

\section{INTRODUCTION}

In recent years of rapid development, there has been drastic increase in demand for electricity resulting in expansion of system size thereby increasing number of generators leading to complexity in the power system. In order to meet this demand a reliable and economic electric power supply is obligatory. The electricity utility companies providing this power supply are faced with many challenges, one of which is generator maintenance scheduling (GMS), which if inappropriate directly affects the reliability and overall system cost. The major goal of GMS is allocation of generating units effectively in order to avoid premature ageing and failure of generators and ensuring maximum system reliability, minimising production cost, extending generator life span subject to some unit and system constraints.

Numerous mathematical and heuristic techniques have been employed so far to solve the GMS. Earlier, mathematical methods such as branch and bound techniques, dynamic and integer programming were applied which gave exact optimal solution for small problems. On a large scale as complexity of systems increased, the number of combination of states increased exponentially and it became computationally prohibitive. In order to overcome these limitations, new concepts have emerged in recent years. They include genetic algorithm (GA), simulated annealing (SA), evolutionary programming, fuzzy logic and their hybrids. The application of GA, SA and hybrid GA/SA to GMS presented in [1] has been compared with each other. This led to an idea of applying particle swarm optimisation (PSO) technique to GMS problem similar to the one presented in [1]. Thus study and application of PSO technique to GMS constitutes the main focus of this paper.

In this paper we study a particle swarm optimisation technique for solving the GMS problem.PSO was introduced by Kennedy and Eberhart in 1995 [2] as an alternative to GA. This technique has capability of simultaneous searching, fast convergence and conserving the past record related to maintenance scheduling hence can be applied to the given GMS problem undoubtedly.

\section{PROBLEM FORMULATION}

Appropriate management and effective scheduling of generator units for purpose of maintenance economically and reliably is the objective function of this problem. In order to make GMS feasible it is important to fulfil certain basic constraints which are as follows [3]:

Maintenance window and sequence constraints -Each unit has an outage for maintenance. It sets maintenance timetable. It states the start and finish of the maintenance at the beginning and the end of the particular interval respectively.

$>$ Crew and resource constraints - It defines the availability and limits of crew and resources respectively.

$>$ Load and spinning reserve constraints - It indicates that total capacity of all running units at particular interval should not drop below predicted load at that interval.

The objective function to be minimised is given by (1) subject to constraints given by (2)-(4) [3].

$\min _{X_{i t}}\left\{\sum_{t}\left(\sum_{i} P_{i t}-\sum_{i \in I_{t}} \sum_{k \in S_{i t}} X_{i k} \cdot P_{i k}-L_{t}\right)^{2}\right\}$

subject to the maintenance

$$
\sum_{t \in T_{i}} X_{i t}=1 \quad \forall_{i}
$$

the crew constraint

$\sum_{i \in T_{t}} \sum_{k \in S_{i t}} X_{i k} \cdot M_{i k} \leq A M_{t} \forall_{t}$

and the load constraint

$\sum_{i} P_{i t}-\sum_{i \in I_{t}} \sum_{k \in S_{i t}} X_{i k} \cdot P_{i k} \geq L_{t} \forall_{t}$ 
Penalty cost given by (5) is added to the objective function given in (1) if the schedule does not satisfy the above constraints given by (2)-(4).

$$
\begin{aligned}
& \text { Penalty cost }=\sum_{c=1}^{3} w_{c}\left|V_{c}\right| \\
& =w_{1}\left|V_{1}\right|+w_{2}\left|V_{2}\right|+w_{3}\left|V_{3}\right|
\end{aligned}
$$

where,

1. $\left|V_{1}\right|,\left|V_{2}\right|$ and $\left|V_{3}\right|$ are amount of violations of maintenance window, crew and load constraints, respectively $\left|V_{c}\right|$ amount of violation of constraint $\mathrm{c}$.

2. $w_{1}, w_{2}$ and $w_{3}$ weighting coefficients of maintenance window, crew and load constraints, respectively.

3. $w_{c}$ is weighting coefficient

4. $\mathrm{i}$ is index of generating units and $\mathrm{k}$ is discrete time step

5. $t$ is index of period and $\mathrm{T}$ set of indices of periods in planning horizon.

6. $\mathrm{M}_{\mathrm{it}}$ manpower needed by unit $\mathrm{i}$ at period $\mathrm{t}$.

7. $X_{\text {id }}$ ith particle position in dimension $d$.

8. $P_{\text {it }}$ generating capacity of unit $i$ in period $t$.

9. $\mathrm{L}_{t}$ anticipated load demand for period $t$.

\section{III.PARTICLE SWARM OPTIMIZATION}

\section{A. Introduction}

Particle swarm optimization is a technique based on graceful motion of swarm of birds searching for most fertile feeding location. It is an evolutionary computation technique developed by Kennedy and Eberhert [4]. It is a derivative free algorithm with less parameters with an ability to handle stochastic natured objective functions. Number of particles together constitutes a swarm wandering in search of best solution. Each particle adjusts its velocity and position according to its own flying experience as well as flying experience of other particles present in the swarm.

\section{B. Components of PSO}

The various components of PSO are explained below.

1.Generation of particles' positions and velocities and finding the gbest and pbest :

Each particle traces its own coordinates in the problem space which is associated with the best solution achieved so far and is named as 'pbest'. Similarly, 'gbest' is the best solution obtained so far by any other neighbouring particle in the swarm.

2. Updating particles' position :

After finding the two best values, the particle updates its velocity and position in order to bring it towards its 'gbest' and 'pbest' locations by the following Equations:

\section{PSO velocity update}

[][]$=w \times[][]+2 \times \operatorname{rand}() \times\{$ pbest [][]$-$ $\operatorname{present}[][]\}+2 \times \operatorname{rand}() \times\{\operatorname{pbest}[][$ gbest $]-\operatorname{present}[$ ][ ] $\}$

\section{PSO position update}

$$
\text { present }[][]=\operatorname{present}[][]+\mathrm{v}[][]
$$

The first square bracket represents the dimension and second bracket represents the index of the particle. ' $v$ ' and ' $w$ ' represents current velocity of the particle and inertia weight respectively. The random numbers are generated in range $[0,1]$.
3. Termination Criteria :

The above procedure is repeated until a criterion is met.

\section{PSO Algorithm}

1. For each particle, set particle with possible random number.

2. For each particle, calculate the fitness value. If a better fitness value is obtained than a past best fitness value, update that (past) value with new achieved pbest value.

3. Select a particle with the best fitness value of all the particles as the gbest.

4. For each particle, calculate particle velocity and particle position according to velocity update equation and position update equation respectively.

5. Repeat the above steps until the criterion is met.

\section{PSO flowchart}

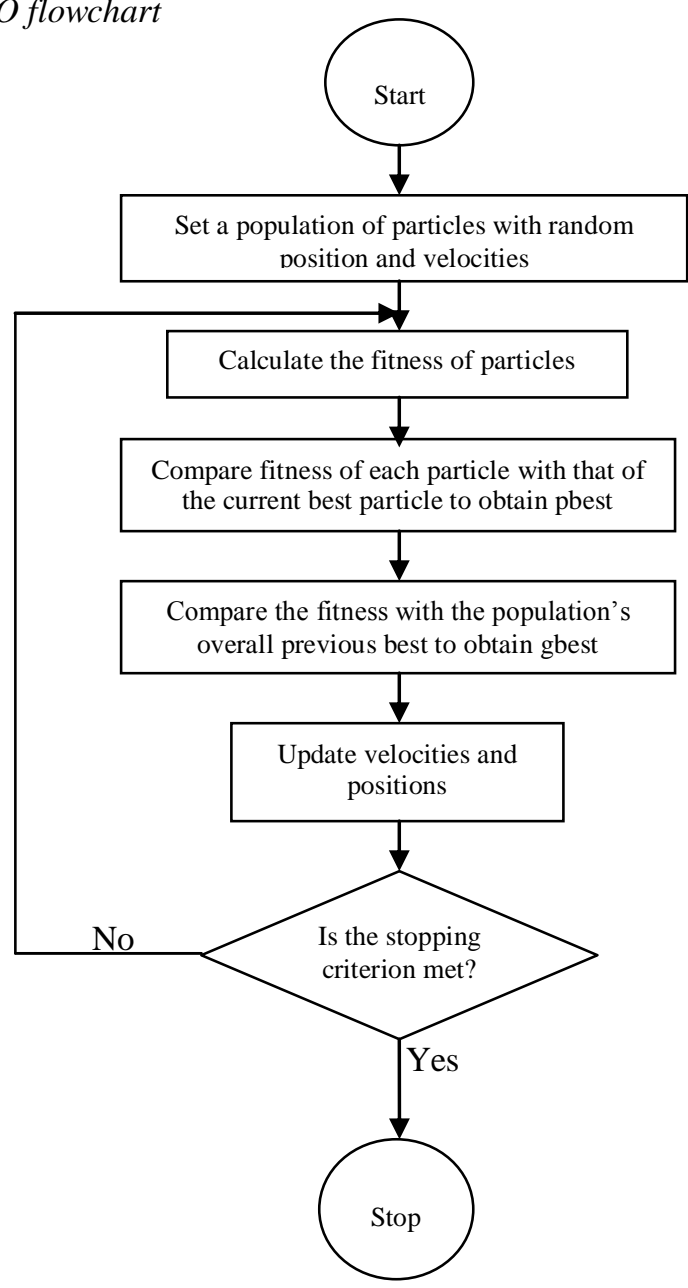

IV.CONCLUSION

For small scale GMS problems mathematical analysis can be carried out. But when a problem is of a large scale, as in any complex system then approaching mathematical analysis becomes difficult. Also some of non-traditional optimization techniques fail to converge and provide optimal solution. However, in PSO each particle is updated based on global optimal point discovered so far. The swarming effect allows the swarm to quickly converge into optimal solution. Hence it is the most time efficient method as compared to other non-traditional optimization methods 
INTERNATIONAL JOURNAL OF INNOVATIVE RESEARCH IN ELECTRICAL, ELECTRONICS, INSTRUMENTATION AND CONTROL ENGINEERING Vol. 4, Issue 2, February 2016

\section{REFERENCES}

[1] Generator maintenance scheduling in power systems using metaheuristic-based hybrid approaches Keshav P. Dahal a,*, Nopasit Chakpitak ba School of Informatics, University of Bradford, Bradford, UK b College of Arts, Media and Technology, Chiang Mai University, Chiang Mai, Thailand Received 17 May 2005; received in revised form 27 February 2006; accepted 30 June 2006 .

[2] J. Kennedy and R. C. Eberhart, "A Discrete Binary Version of the Particle Swarm Algorithm", International Conference on Systems, Man, and Cybernetics, Vol.5, 12-15, pp. 4104 4108, Oct 1997

[3] Y. Yare, G.K. Venayagamoorthy, U.O. Aliyu, " Optimal generator maintenance scheduling using a modified discrete PSO", IET Generation, Transmission \& Distribution.

[4] Kennedy J, Eberhart RC (1995) "Particle swarm optimization." Proceedings IEEE International.

[5] Keshav P. Dahal and Nopasit Chakpitak, "Generator maintenance scheduling in power systems using metaheuristic-based hybrid approaches",2006

[6] A. P. Engelbrecht, Fundamental of Computational Swarm Inteligent, First ed. The atrium, Southern Gate, Chichester, West Sussex PO19 8SQ, England: John Wiley \& Sons Ltd, 2005.

[7] David L. Cushman , "A Particle Swarm Approach to Constrained Optimization Informed by “Global Worst"”,2007 "PDCA12-70 data sheet," Opto Speed SA, Mezzovico, Switzerland.

[8] M. B. Ghalia, "Particle Swarm Optimization with an Improved Exploration-Exploitation Balance," iEEE, vol. 978-1-4244-21671/08/\$25.00 @2008 IEEE, 2008.

[9] Marwali M.K.C., Shahidepour S.M.: 'Coordination between longterm and short-term generation scheduling with network constraints', IEEE Trans Power Syst., 2000, 15, pp. 1161-1167. 